\title{
Aperçu sur les pratiques sacrificielles chez les Mitsogho
}

Mythes et rites initiatiques

A glance at sacrificial practices among the Mitsogho: Myths and rites of initiation

\section{Otto Gollnhofer et Roger Sillans}

\section{OpenEdition}

\section{Journals}

Édition électronique

URL : http://journals.openedition.org/span/466

DOI : $10.4000 /$ span.466

ISSN : 2268-1558

\section{Éditeur}

École pratique des hautes études. Sciences humaines

\section{Édition imprimée}

Date de publication : 1 septembre 1979

Pagination : 167-174

ISSN : 0294-7080

\section{Référence électronique}

Otto Gollnhofer et Roger Sillans, «Aperçu sur les pratiques sacrificielles chez les Mitsogho », Systèmes de pensée en Afrique noire [En ligne], 4 | 1979, mis en ligne le 04 juin 2013, consulté le 30 avril 2019. URL : http://journals.openedition.org/span/466 ; DOI : 10.4000/span.466 


\author{
A P E R G U S U R L E S P R A T I Q U E S \\ S A C R I F I C I E L L E S C H E Z L E S M I T S O G H O \\ Mythes et rites initiatiques \\ par Otto Gozzhofer et Roger Sizzans
}

Les pratiques sacrificielles chez les Mitsogho du Gabon central, intimement liêes à la notion de sacré, peuvent être appréhendées à plusieurs niveaux : d'une part, celui de la magie et de la sorcellerie, d'autre part, celui des rituels qui tendent à réactualiser le sacrifice primordial de deux créatures mythiques. Il s'agit de Dinzona (1'une des deux épouses de Kombe, le soleil, chef du village des désincarnés) (1) et de Ya Mwei, le génie de l'eau (2). Nous verrons que les rites liês à ces deux puissances s'inscrivent dans le cadre de deux sociétés initiatiques masculines, la société du Bwete (pour Dinzona) (3) et celle du Ya Mwei (pour le génie de l'eau).

La notion de sacré qui se dégage du terme maghanga (4), couvre toute manipulation occulte (bénéfique ou maléfique) ou purement symbolique. Ce terme est équivalent à celui de bogha, mais il s'emploie dans un contexte diffêrent. Car ce dernier terme dësigne toute

1. Sillans, R., Motombi. Récits et énigmes initiatiques des Mitsogho du Gabon central. I. La route de la vie, Thèse de IIle cycle, Paris, Institut d'ethnologie, 1971 (Coll. Archives et documents, microfiche $\left.n^{0} \cdot 71-00-21\right)$.

2. Gollnhofer, 0 , et Sillans, R., Le mythe de la découverte du génie de 1 'eau chez les Mitsogho, L'Ethnographie (sous presse).

3. Gollnhofer, 0., Les rites de passage de la société initiatique du Bwete chez les Mitsogho. La manducation de Z'iboga, Thèse de IIIe cycle, Paris, Université René Descartes, 1974.

4. Gollnhofer, O. et Sillans, R., Cadres, élêments et techniques de la médecine traditionnelie tsogho. Aspects psychothérapiques, Esychopathologie africaine, XI, 3, 1975, 285-321. 
médication à base de substances naturelles ou chimiques, tandis que le premier se rapporte à l'êtat psychique et spirituel de l'être. Ces deux sortes de médications visant à un rétablissement de l'êquilibre psychique, somatique ou psychosomatique, les Mitsogho les désignent en français par le terme "médicament".

Les pratiques sacrificielles font appel à des entités mythiques ou ancestrales. On les invoque pour obtenir leur concours sous des formes multiples. Mais, quelle que soit la nature de l'invocation (bénéfique ou maléfique), tous les rites de type sacrificiel tendent à réaliser la jonction de deux plans d'existence : celui des désincarnés, dans le monde supra-physique et celui des réincarnés, dans le monde physique.

En effet, ces deux mondes -- selon le concept tsogho -- n'en font qu'un (5). Ils se traduisent concrètement, pour l'homme, par le changement et le mouvement incessant entre le "pays natal" (1'au-delà) et le "campement" (1a terre), entre lesquels 1 'homme effectue un va-etvient : "nous venons, nous retournons".

Dans cette dimension de l'unicité du monde, la vie terrestre est considérée comme une étape inférieure, conçue comme une chute de l'être en l'état où il est revêtu d'une forme.

L'homme se réfère à son passé immatériel par l'intermédiaire de la chaîne des entités spirituelles sollicitées. Il vise à acquérir une faculté qui n'est à la disposition des vivants que par des moyens rituels adéquats. Il devient, alors, un manipulateur du sacré en faisant agir des forces supërieures à lui mais qui demeurent, toutefois, sous son contrôle et en sa faveur, temporairement néanmoins.

En vue de réaliser la jonction des deux plans d'existence, on fait intervenir soit des supports de symboles -- dans le contenu desquels entrent des éléments d'origine humaine, animale, végétale et minérale (6) -- soit des pratiques sacrificielles.

5. Gollnhofer O. et Sillans R., Recherche sur le mysticisme des Mitsogho, peuple de montagnards du Gabon central (Afrique équatoriale), in : Réincamation et vie mystique en Afmique noire, Paris, PUF, 1965, pp. 143-177.

6. Gollnhofer 0, ex Sillans R., Guérisseurs d'Afrique noire, Médecine de Z'Horme, $\mathrm{n}^{\circ} 41$, janv. 1972, pp. 30-33. En ce qui concerne certains objets supports de symboles, comme les objets-médiateurs du 
Comme pour l'intervention d'un support de symboles (7), tout acte sacrificiel, individuel ou collectif, est toujours accompagné de paroles et de récits qui lui assignent sa destination.

D'après le mythe sur les origines de la sociêté du Bwete, l'initiation en ses dêbuts impliquait nécessairement le sacrifice d'une entitê spirituelle, celui de Dinzona. C'était une fenme lumineuse et de grande beauté, rivale de Ngonde, la lune, seconde épouse de Kombe, le soleil. Par suite de l'hostilité de sa rivale Ngonde, Dinzona, bien qu'ètant la prêférêe de Kombe, dut quitter son village de l'au-delà et se vit condamnée à descendre sur terre. C'est alors qu'elle transmigra dans l'un des plus beaux et très grands copaliers, à êcorce rouge, de la forêt équatoriale, l'arbre Motonbi, qui est devenu L'arbre de vie des Mitsogho, au pied duquel a lieu la deuxième partie des rites de passage de la société initiatique du Bwete.

Autrefois, au début des rites de l'initiation, aux premiers jours de la manducation de l'iboga (8), on abattait le Motombi, "on le faisait coucher" -- comme le disent les initiés -- pour simuler le sacrifice de Dinzona qui est aussi celui du rëcipiendaire. Par les rites que lui font subir les anciens initiés, ce dernier, en effet, est censé entrer dans une phase d'inexistence, son esprit quittant 1e corps pour entreprendre un voyage mystique (9). Voyage au cours duquel il se rend, après de multiples péripéties, au "village de kombe" et converse avec diverses entités spirituelles telles que Kombe lui-même (le soleil), Ngonde (la lune) er Minanga (les étoiles). Il rencontre également ses ancêtres quí lui posent, à tour de rôle, la question : "Qu'est-tu venu faire ici ?" Question à laquelle le récipiendaire répond : "Je suis venu voir le Bwete !" Et les ancêtres de répondre : "...Le Bwete, c'est nous tes ancêtres !" Puis ils interrogent le

type mbumba, etc, se reporter à l'ouvrage de Gollrhofer O., Sallêe,P. et Sillans, $\mathrm{n}$., Art et artisanat tsogho, paris, 1975 (Travaux et documents de I'OnSTOM, n 42 ).

7. Gollnhofer, 0. et Sillans, R., Symbolisme et prophylaxie chez les Mitsogho (Gabon, Anthropos, 73, 1978, 449-460.

8. Tabernanthe iboga Baillon (Apocynacées). Voir aussi Gollnhofer 0 . et Sillans R., L'usage religieux des stupéfiants, Médecine de 2'Homme, $\mathrm{n}^{\circ} 49$, nov. 1972, 26-31.

9. Go1lnhofer 0. et Sillans R., Aspects phénoménologiques et initiatiques de l'état de destructuration temporaire de la conscience habituelle chez les Mitsogho du Gabon, Bgychopathologie africaine, XII, $1,1976,45-75$. 
visiteur : "Es-tu venu pour toujours ou pour faire une visite ?" Après avoir répondu que ce n'étaít que"pour faire une visite", l'esprit du néophyte retourne sur terre et réintégre son corps.

En fait, la séparation de l'esprit du postulant de son corps équivaut à un rituel de mise à mort de type sacrificiel. Jusqu'à l'obtention de la vision normative du Bwete, les postulants doivent obligatoirement s'installer dans le côté gauche de la maison de culte ou ebanza, lequel est, du point de vue symbolique, le domaine de la mort (10).

Mais le principal rite réactualisant le sacrifice primordial est 1ui-même en rapport avec un sacrifice humain. Ainsi que nous 1 'avons dit, on abattait ou, plus exactement, on faisait "coucher le Motombi", ce qui signifiait la mort de Dinzona et celle des néophytes. Car les arbres, dans la pensée symbolique tsogho, sont considérés comme des personnes. Aussi, est-ce la raison pour laquelle, au moment du décès, on coupe un arbre symbolisant le défunt.

Pour "rearesser le Motombi" et, ainsi, faire revenir les néophytes de la mort symbolique, on procédait à un sacrifice humain qui consistait dans la mise à mort rituelle de deux jeunes filles. Cette pratique sacrificielle est reproduite de nos jours sur le plan symbolique au cours d'un rite où trois personnages apparaissent. Deux initiés travestis miment les jeunes filles qu'un homme menace sans cesse avec un sabre de bois.

I1 faut reconnâtre, en effet, qu'autrefois on se livrait fréquemment à ce genre de sacrifices humains, en cas de guerres aves les populations avoisinantes. Lorsqu'un telle éventualité se présentait, chaque clan décidait de fabriquer des mikuku, terme signifiant "malin, rusé", c'est à dire des talismans prophylactiques "pour se rendre invisibles et invulnérables au combat" (11). Leur préparation exigeait le sacrifice de deux jeunes filles vierges dont le sang

10. Gollnhofer O. et Sillans R., Le symbolisme chez les Mitsogho. Aspects de I'anthropomorphisme dans la société initiatique du Bwete, in Systèmes de signes. Hommage à Germaine Dieterlen, Paris, Hermann, 1978, pp. 223-241.

11. Gollnhofer 0., Bokudu. Ethno-histuire ghetsogho. Tome I Essai sur l'histoire générale de la tribu d'après la tradition oraze, Diplome de l'E.P.H.E. (Ve Section), Paris, Institut d'ethnologie, 1972 (Co11. Archives et documents, microfiche $\mathrm{n}^{\circ}$ 71-00-20). 
mélangê aux cendres de leur corps incinéré devait être appliqué sur celui des guerriers mitsogho. Par ce sacrifice rituel, chacune des personnes sacrifiées devenait alors, pour chaque clan, une entité spirituelle dénonmée kono. Ces entitês font partie de la catégorie de remèdes spirituels dits maghangha ou "médicaments", comme nous 1 'avons précédemment exposé.

Au cours de la vision qu'ont les récipiendaires du Bwete, les mikuku leur apparaissent sous des formes fugaces comme autant d'imageries hypnagogiques qui leur font éprouver de vives craintes.

Cet usage du sacrifice humain, dans un but prophylactique, était très courant autrefois pour se protéger contre toute intervention maléfique, éviter des épidémies et le retour de malheurs déjà survenus. Il pouvait revêtir les aspects les plus divers. C'est ainsi que la communauté villageoise décidait de déterrer un cadavre afin d'en conserver les ossements, surtout le crâne, dans un endroit spécial, au dépôt des objets rituels, dénommé la "maison des médicaments". Toutefois, préalablement à 1 'exhumation, on précisait au cadavre dans quel but on allait le déterrer. Mais pour que cette protection désirée pût être effective, "il fallait qu'un autre, du même clan, prît sa place au cimetière", ce qui impliquait un sacrifice humain.

Nous devons aussi mentionner 1 'auto-sacrifice des grands initiés de la société du Bwete. En l'état actuel de nos connaissances, il est difficile d'affirmer s'il s'agit d'un fait historique ou s'il se produit encore de nos jours. L'initié, toujours une personne âgée, prédisait le jour de sa mort, souvent des années à l'avance (12). A l'approche de cette date, tous les membres de la société du Bwete se réunissaient dans la maison de culte pour se livrer aux rites du Bwete de mort suivis de ceux de deuil. Rites au cours desquels l'homme qui avait désiré mourir dans un cadre rituel avec des funérailles bien déterminées, succombait inéluctablement par le phénomène de la mort psychogène. Il ne restait plus qu'à transporter son cadavre et à l'enterrer au pied de l'arbre Motombi, ainsi que le veut la tradition pour tous les chefs religieux de cette société initiatique,

12. Gollhofer 0 . et Sillans R., Aspects du phénomènes de consensus dans la psychothérapie ghetsogho, in La notion de personne en Afrique noire, Paris, C.N.R.S., 1973, pp. 545-563. 
ou le conserver, pour l'usage prophylactique, dans le dêpôt des accessoires rituels, après des techniques appropriées de thanatopraxie (13).

Les rites de mort et de devil sont, d'ailleurs, pour tous les initiés, identiques, sauf dans certains cas bien déterminés. Ces rites funèbres sont d'une importance capitale dans le symbolisme tsogho, car c'est par eux qu'on guide l'esprit du défunt. Et c'est surtout pour cette raison que les rites d'initiation sont pratiquement identiques à eux. I1 faut, en effet, envoyer l'esprit du défunt à la source, au "pays natal", à cet au-delà dont il est issu, afin qu'il ne s'égare. Autrement dit, il faut lui faire recouvrer son existence initiale, imnatêrielle, en attendant sa prochaine désincarnation. Ce sont donc ses deuxièmes funérailles par rapport à l'initiation au cours de laquelle, dans un contexte sacrificiel, 1 'initiê a dẻjà rituellement subi sa propre mort.

Nous avons, jusqu'à présent, fait état d'un seul sacrifice primordial, celui relevant de lá société initiatique du Bwete, c'est à dire l'ancêtre élevé du cađre lignager au cadre tribal collectif. Nous allons maintenant 1 'aborder dans le cadre de la sociêté du Ya Mwei, le génie de l'eau.

Selon le mythe de Ya Mwei, ce génie a été. découvert au cours d'une partie de pêche, par les femmes, dans une mare. Il avait avalé trois de ces femmes; les autres avaient demandé du secours aux hommes afin de les aider et de faire sortir cette "Chose". de l'eau. Les hommes se 1'approprièrent et 1 'amenèrent au village, car Ya Mwei êtait une "Chose", et ils lui construisirent une cabane de feuillages. Mais lorsque la grande saison sèche arriva, Ya Mwei mourut à cause de la grande chaleur. I'un des hommes, nommé Dikumbu, avait bu du sang de Ya Mwei et imita sa voix caverneuse. Aussitôt les hommes se mirent en colère, reprochant à Dikumbu de s'être substitué à Ya Mwei et, en conséquence, d'avoir dévoilé la mort du génie. Alors Dikumbu avait êté mis à mort non sans avoir, au prêalable, initié quelques-uns des hommes. Depuis ce moment, les initiés doivent jurer, solennellement,

13. Gollnhofer O. et Sillans R., Thanatopraxie et inhumation chez les Mitsogho, L'ethnographie 'sous presse). 
de ne pas dévoiler 1 'identité de Ya Mwei, de ne jamais dire que Ya Mwei est une personne, mais qu'il est une Chose. Dans le cas contraire, la punition est la mort (14). Car Ya Mwei demeure vivant et est perpétué par ses enfants, les mbuna, autrement dit les initiês qu'il met au monde. Son corps entra sous terre et son esprit retourna dans 1 'eau.

Ce résumé du mythe de Ya Mwei nous fait apparâtre un triple sacrifice. Celui du génie, perpétré par Kombe (le soleil), suivi de la mise à mort de Dikumbu par les hommes et finalement la mort symbolique des néophytes qui seront "avalês" par Ya Mwei et ensuite "accouchés" au monde initiatique au cours de chaque rite de passage. L'initiation au Ya Mwei est la préfiguration de l'enterrement futur de l'initié et aussi sa résurrection. En effet, au début des rites de passage, les aspirants sont frottés au kaolin. Puis on les conduit, suivant un trajet comprenant neuf barrages en feuilles, jusqu'à la hutte où est dissimulé Ya Mwei. A cet endroit, on fait baigner les néophytes dans un trou, pratiqué dans une terre argileuse, empli d'eau, de silures, de crabes et de têtards ; ce trou correspond au vagin eneso de Ya Mwei. Quant à l'argile mouillée dont est badigeonné le corps des récipiendaires, elle signifie "qu'ils ont bien été avalés par Ya Mwei", car ils ont toutes les saletés sur leurs corps comme les enfants dans la matrice. Les mbuna, muets, courbant le corps, baissant les yeux et la tête, croisant les bras derrière le dos -- "marquant ainsi qu'ils n'ont encore ni bras ni jambes" -sont ensuite conduits au village. Là, on les fait asseoir à même la terre sous le soleił brûlant comme était Ya Mwei avant de mourir à la vie physique et renâtre à la vie spirituelle. Tout comme renâ̂tront les récipendaires "accouchês" par Ya Mwei, lequel, dissimulé dans la brousse, près du village, pourra s'écrier : "J'ai mis au monde mes enfants, je retourne ; gardez-les bien !"

Bien que nous n'ayons fait état, dans cette étude, que des pratiques sacrificielles dans leurs rapports avec les mythes et les rites initiatiques, nous n'hésitons pas à affirmer que chez les

14. Gollnhofer 0 . et Sillans R., Tsâmbo, texte rituel de guérison chez les Mitsogho, L'ethnographie, 1, 1978, pp. 45-53. 
Mitsogho ces pratiques sont au premier plan des faits rituels.

$\mathrm{Si}$, dans cette ethnie, le sacrifice se manifeste, indiscutablement, d'une manière beaucoup moins ostensible que dans la plupart des ethnies non bantu, cela tient au fait que cette pratique rituelle est, dans 1 'ensemble, moins un acte rẻel et prépondérant dans la vie religieuse qu'une ëmergence du symbolisme des mythes. 